\title{
Development and Application of an Integrated Approach toward NASA Airspace Systems Research
}

\author{
Richard Barhydt ${ }^{1}$ and Robert K. Fong ${ }^{2}$ \\ National Aeronautics and Space Administration \\ Paul D. Abramson ${ }^{3}$ \\ PDA Associates \\ and \\ Ed Koenke \\ Genasys Consulting Services, Inc.
}

\begin{abstract}
The National Aeronautics and Space Administration's (NASA) Airspace Systems Program is contributing air traffic management research in support of the 2025 Next Generation Air Transportation System (NextGen). Contributions support research and development needs provided by the interagency Joint Planning and Development Office (JPDO). These needs generally call for integrated technical solutions that improve systemlevel performance and work effectively across multiple domains and planning time horizons. In response, the Airspace Systems Program is pursuing an integrated research approach and has adapted systems engineering best practices for application in a research environment. Systems engineering methods aim to enable researchers to methodically compare different technical approaches, consider system-level performance, and develop compatible solutions. Systems engineering activities are performed iteratively as the research matures. Products of this approach include a demand and needs analysis, system-level descriptions focusing on NASA research contributions, system assessment and design studies, and common systemlevel metrics, scenarios, and assumptions. Results from the first systems engineering iteration include a preliminary demand and needs analysis; a functional modeling tool; and initial system-level metrics, scenario characteristics, and assumptions. Demand and needs analysis results suggest that several advanced concepts can mitigate demand/capacity imbalances for NextGen, but fall short of enabling three-times current-day capacity at the nation's busiest airports and airspace. Current activities are focusing on standardizing metrics, scenarios, and assumptions, conducting system-level performance assessments of integrated research solutions, and exploring key system design interfaces.
\end{abstract}

\section{Introduction}

Air traffic delays reached record levels in 2007. ${ }^{1}$ Compounding this problem, demand for air travel in the U.S. continues to grow at rates greater than the current air traffic system can accommodate. ${ }^{2}$ This demand growth is significant enough to require major and fundamental changes to the National Airspace System (NAS). The interagency Joint Planning and Development Office (JPDO) is addressing these needs by developing the vision for a 2025 Next Generation Air Transportation System (NextGen) and defining the research required to achieve that vision. $^{2}$ As one of the JPDO member agencies, the National Aeronautics and Space Administration (NASA) is

\footnotetext{
${ }^{1}$ Project Scientist, NASA NextGen Airspace Project, NASA Langley Research Center MS 152 Hampton, VA 23681, Member.

2 Associate Principal Investigator for System-Level Design, Analysis, and Simulation Tools, NASA NextGen Airspace Project, NASA Ames Research Center Moffett Field, CA 94035.

${ }^{3}$ Principal, 4 Hampshire Road, Wayland, MA, 01778, Senior Member.

${ }^{4}$ President, 420 Highland Drive, Mays Landing, NJ, 08330.
} 
conducting long-term research needed to support the NextGen vision. As part of this effort, the NASA Airspace Systems Program contributes revolutionary air traffic management (ATM) concepts and technologies.

JPDO research partners provide the knowledge needed to support informed regulatory and investment decisions for NextGen. ${ }^{3}$ NASA contributes research results in response to specific JPDO research and development needs. ${ }^{3}$ These needs typically call for research contributions that comparatively assess the system-level impact of multiple technical approaches. They generally also request integrated solutions across a range of operational domains, planning time horizons, and ATM sub-systems. The combined requirements for multi-solution comparison, systemwide performance improvement, and complex system compatibility necessitate an integrated research approach.

Other organizations facing similar challenges have provided a good foundation for structured, integrated research. Leveraging a "Validation Data Repository" originally developed by Eurocontrol, the FAA has applied conclusions drawn from assimilated results toward research investment decisions. ${ }^{4,5,6}$ This collaborative work between the FAA and Eurocontrol has established sound fundamentals for comparing research results, including the need to apply common definitions, scenarios, metrics, and assumptions. Recognizing the need for operational concepts that improve system-level performance, MITRE has explored system-level trade-offs across a two-factor concept space. ${ }^{7}$ In a case study, concept variations were represented by a continuous variable that explicitly defined system-level performance. This work provided an effective means for depicting system-level performance across a range of operational concepts.

In developing its research integration approach, NASA is applying these principles and building upon work begun previously in the Virtual Airspace Modeling and Simulation (VAMS) Project. ${ }^{8}$ Under the VAMS Project, NASA developed methods and tools for assimilating research concepts and assessing their contributions toward system-level performance. Within the Airspace Systems Program, NASA is expanding these capabilities to deliver integrated research solutions to the JPDO. A system-level research group facilitates delivery of integrated solutions by providing the following products: system-level operational, functional, and technical descriptions that relate the research results in a system-wide, gate-to-gate context; common system-level metrics, scenarios, and assumptions; assessments that evaluate the research products' contribution to system-level performance; design studies that establish sub-system compatibility; and system-level models that support the assessments and design studies. These products are developed iteratively and are periodically documented in an internal Technical Integration Report. This report focuses on research products developed by NASA and does not aim to provide a comprehensive NextGen design, as JPDO has done with its Enterprise Architecture. ${ }^{9}$ The NASA Airspace Systems Program has completed a baseline Technical Integration Report that will be refined as the research matures. The following sections discuss how these products are produced and how they can enable the program to deliver integrated research results. Highlights from the baseline Technical Integration Report, current integration activities, and challenges faced are also discussed.

\section{Systems Engineering Approach to Research Integration}

Research integration methods must support the program's need to compare technical solutions and develop capabilities that will interact well and improve overall system performance.

Studying multiple technical alternatives provides several advantages. Alternative solutions may produce more optimal performance under different operating conditions. Also, NextGen decision makers and implementers will certainly apply additional performance criteria such as cost that are not directly addressed by NASA. Airspace Systems research therefore emphasizes trade studies that comparatively evaluate the performance of different proposed technical solutions. Alternatives considered often exhibit different levels of a common attribute, such as the degree of authority assigned to air and ground systems or humans and automation. This range of conditions is referred to hereafter as a "trade space". Research contributions supporting strategic NextGen decisions require the careful comparison of results from multiple studies investigating different areas of a defined trade space.

As is the case in the current NAS, NextGen is a tremendously complicated, interconnecting set of systems, each substantial in its own right. NASA Airspace Systems research is studying key system improvements spanning a gate-to-gate flight environment. Across this diverse range of systems and domains, the JPDO is proposing a number of significant changes to air and ground technologies and procedures. ${ }^{2}$ New capabilities such as trajectory-based operations and performance-based services will rely on greater levels of precision, coordination, and information processing. Because demand/capacity imbalances exist throughout the NAS, the JPDO envisions these capabilities being implemented across flight domains ranging from surface operations to en route airspace. ${ }^{3} \quad$ Techniques applied in one domain will be highly interdependent with those in another. Full benefits can therefore only be 
realized by striving for concepts and technologies that provide seamless transitions across flight domains and ATM functions.

To effectively manage this complex structure, Federal Aviation Administration (FAA) acquisition and operational practices have relied heavily on systems engineering. ${ }^{10}$ Practitioners of systems engineering seek to achieve a desired system performance through effective consideration of internal and external interactions and constraints. The FAA uses systems engineering to study sub-system interactions and assess system-level performance of proposed technologies. ${ }^{11}$ The JPDO is using systems engineering to manage its Enterprise Architecture and expects that partner organizations will also use systems engineering methods to manage their own processes. $^{3}$

NASA's desire to explore alternative technical solutions that improve overall system performance suggests a strong benefit from applying systems engineering principles to its airspace systems research. Systems engineering supports development of the integration research products listed above. Although systems engineering is often applied as part of a hardware or software development activity, its application in a research program appears to be less common.

The systems engineering framework enables more effective technical communication with external stakeholders and within the research team. A major part of this framework is a set of three complementary system-wide descriptions: operational, functional, and technical. The descriptions represent different viewpoints of the overall system. Concept elements are connected to show how flights would operate in a gate-to-gate environment. These concepts can be decomposed into functions that offer insight into the system architecture. Technical solutions refer to advanced capabilities that support the concepts and functions. They include tools for cataloguing, predicting, and using information; algorithms, and detailed internal and external interface descriptions.

Both system-level and detailed technical researchers contribute to this design framework through top-down and bottom-up communication, respectively. Alternative approaches to needed system-level capabilities are discussed side-by-side, encouraging productive dialogue on ways to address common technical challenges. With a broader perspective, researchers can also generate innovative new solutions that draw from an understanding of relative strengths and weaknesses between those previously evaluated.

Systems engineering can help provide a foundation of common system level metrics, scenarios, and assumptions that enable necessary comparative analyses. In order to ensure meaningful trade space comparisons, different solutions must be evaluated using common performance criteria (metrics) under the same operating conditions (scenarios). Consistent assumptions mitigate the chance that legitimate performance differences between solutions will be confounded by different responses to unequal external factors. Without trade space analyses, contributions to many JPDO research needs will be incomplete.

The systems approach also strengthens research relevance by informing the choice of research activities and delivering solutions that address system-wide performance needs and respect interface constraints. Comparing research results with performance targets can demonstrate that a proposed approach is sufficient to meet the specified need. When the target is reached, further refinement may be unnecessary. Solutions that respect interface constraints will facilitate adaptation to an operational environment. Assessments can indicate the research activities having significant impact on overall system performance. These results can help inform research investment decisions. Systems engineering also provides structure that should assist the Airspace Systems Program in delivering research products at specific points along the NextGen implementation schedule.

\section{Systems Engineering Methodology}

The NASA Airspace Systems Program is tailoring systems engineering best practices ${ }^{10,12,13}$ for application in a research environment. Goals of the integrated research approach supported by systems engineering include:

- Close alignment of NASA research activities with JPDO research needs,

- Ability to compare different concept and technology options,

- Research activities influenced by system-level performance considerations, and

- Exploration of key cross-domain and sub-system interactions.

When developing its research-based systems engineering approach, the NASA Airspace Systems Program leveraged methods used by the FAA, Department of Defense (DoD), and the NASA space program. ${ }^{10,12,13}$ These large organizations successfully use systems engineering for complex system design, development, and acquisition projects.

Systems engineering provides an iterative, structured process typically consisting of requirements analysis, functional analysis, system design and definition, and performance validation. ${ }^{12,14}$ When applied to a product 
development effort, each successive cycle adds greater detail to the system design until a prototype is eventually constructed and evaluated. The systems engineering research approach retains this iterative nature, providing greater definition of various concept solutions as research is conducted. The process maintains several characteristics of a "Concept Development" stage, ${ }^{14}$ without converging on a single solution. The approach covers work in four JPDO-defined functions: Capacity Management, Flow Contingency Management, Trajectory Management, and Separation Management. ${ }^{2}$ Research groups investigate these functions across the surface, terminal area, and en route domains.

Figure 1 shows the major components of the systems engineering approach adapted for ATM research. It maintains a similar structure to Fig. 3-1 in the DoD Systems Engineering Fundamentals handbook. ${ }^{12}$ A demand and needs analysis drives descriptions of the anticipated future NAS from the three points of view (Operational, Functional, and Technical), all of which become better defined as the NASA research matures. Descriptions supporting each view present multiple alternative solutions. To ensure continuous gate-to-gate concept coverage, engineering judgment or limited outside research results are used where needed to fill in necessary details not directly addressed by NASA research. The three descriptions, however, provide much more detailed coverage in concept or technology areas studied by NASA. Collectively, the three views help facilitate system-wide interoperability across the research products.

Development of the three descriptions is supported by systems analysis and system concept assessments. These activities respectively evaluate feasibility and potential benefits for options presented in each view. This entire process is performed iteratively based on updated JPDO guidance, FAA system demand and capacity forecasts, and research progress.

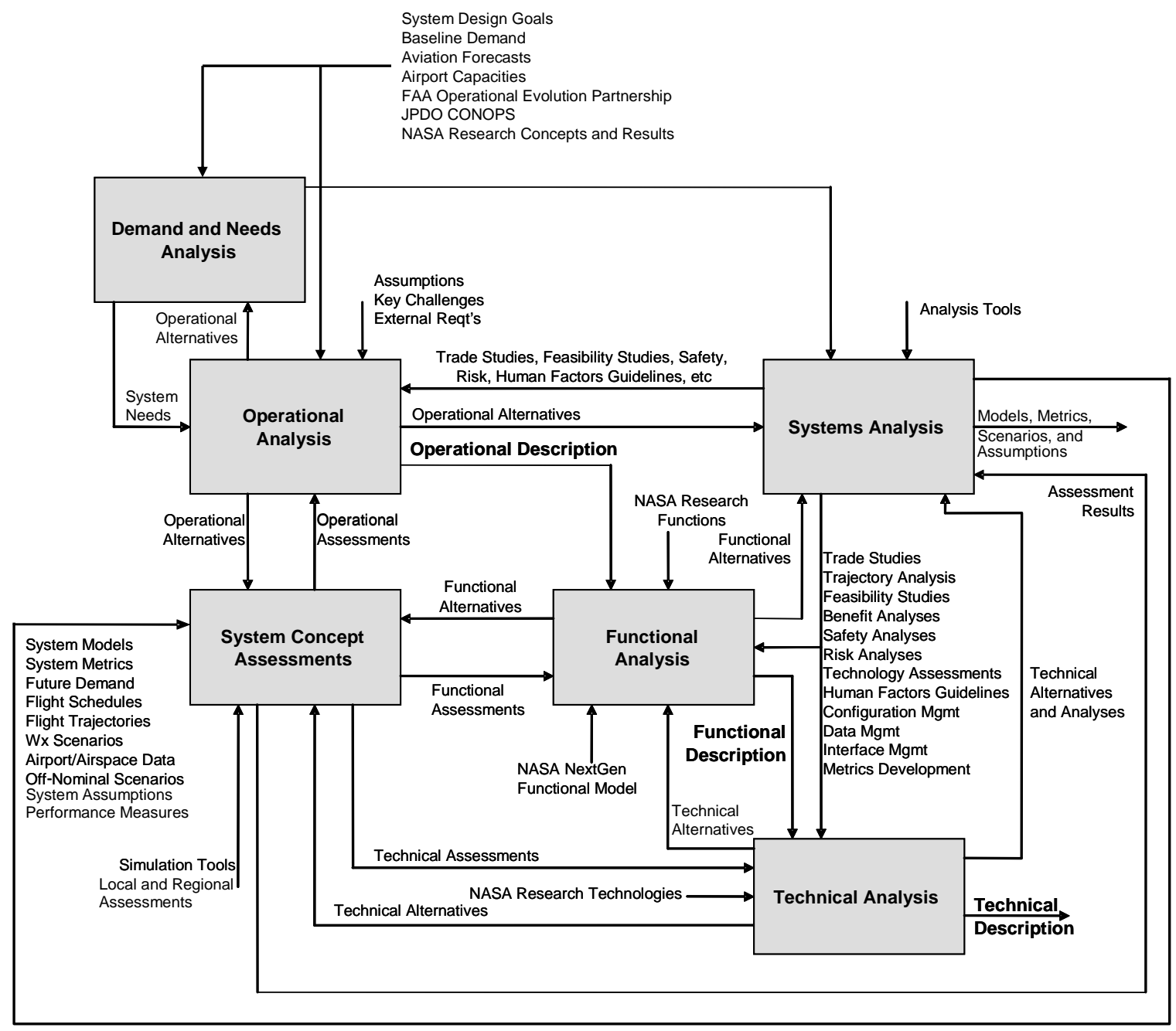

Figure 1 Research-Focused Systems Engineering Process

American Institute of Aeronautics and Astronautics 092407 
The process begins with a demand and needs analysis that estimates future demand for air traffic management (ATM) services based on aviation growth forecasts. The analysis offers an initial assessment of the future system's ability to accommodate the projected demand. Gaps between needs and potential capabilities suggest areas needing research attention.

An operational analysis formulates a description of concept options that are responsive to the system needs identified in the demand and needs analysis. Concept options offer different potential approaches to achieving the operational improvements discussed in the JPDO Integrated Work Plan. ${ }^{3}$ System-wide interactions among concepts and their supporting infrastructure ("enablers") form the basis of the operational description. Possible airspace structures and walk-through scenarios support the concepts and enablers. Scenarios show what it would be like to go through the operation from a flight deck, Air Navigation Service Provider (ANSP), and airline Flight Operations Center (FOC) point of view. Variations on these scenarios may be created for different concept options. In most cases, trade studies and analyses are needed to fully define and evaluate the range of concept options considered.

A functional analysis decomposes the operational concepts into a set of alternative functions (actions and tasks $)^{14}$ and associated functional requirements. The functions and their requirements make up the functional description. The functional analysis allows for a comparison between the NASA-generated functions and those defined by the JPDO NextGen Enterprise Architecture. ${ }^{9}$

A technical analysis incorporates the results of detailed research activities, such as algorithms or information processing tools, conducted across the Airspace Systems Program. The resulting integration forms a technical description that provides a set of technical design alternatives. The technical description includes both a physical description (i.e., the elements that will accomplish the functions such as aircraft avionics, pilot, controller, dispatcher, ground systems, decision support tools, and automation) and the logical description that specifies the information and the data flow among the selected physical elements. The technical description shows how the research results contribute to design of the functions described in the functional description and the operations described in the operational description.

System concept assessments gauge the potential system-wide benefits of different integrated concept solutions and technologies. These assessments support JPDO activities that evaluate the ability of operational improvements to meet NextGen performance targets. Systems analysis provides analytical support to the system concept assessments. The analysis uses environmental and traffic conditions to generate system-level metrics and scenarios used for assessments. It contributes common system-level assumptions in areas including implementation timeframe, information availability, and anticipated enabler capabilities. It also supports feasibility studies across key sub-system interfaces. Technical researchers complement broader system-level studies by conducting local trade studies and assessments. These focused analyses consider the merits of specific technical alternatives within a higher fidelity and more limited domain.

Systems engineering provides and supports a common language that forms a basis against which all program research can be performed. This language includes system-level technical challenges, design assumptions, metrics and performance requirements, airport and airspace data, and nominal and off-nominal traffic and weather scenarios. Applying these elements consistently across the program is a major challenge and focus of the systems engineering research effort.

\section{Application of Systems Engineering Approach to NASA Airspace Systems Research}

The NASA Airspace Systems Program has begun to apply the methodology discussed above to its research. Activities shown in Figure 1 provide an overall framework within which individual research activities contribute to higher level goals established by the JPDO. This framework enables researchers to compare technical results across alternative solutions and consider how those solutions interact in a system-wide context. A system-level research group coordinates the activities shown in Figure 1 and facilitates information exchange between the activities. Through the information exchange, this group influences research activity choices and methods. Researchers in other technical disciplines have a comparable role in developing the system-level descriptions. These researchers offer detailed technical expertise and a strong familiarity with the potential capabilities and limitations of the technologies they develop. Their insight helps reveal detailed sub-system or component behaviors that may impact system-wide performance. Such issues may not surface during broad system-level assessments and require higherfidelity simulation tools that address local or regional issues. These complementary contributions between the system-level and other technical research groups form the basis of the three contributing system-level descriptions.

Information links shown in Figure 1 provide supporting information to connecting activities. The research team, however, does not rigidly follow a sequential flow process. Starting with a general understanding of research needs 
and system requirements, concurrent research efforts support advancement of each Figure 1 activity. As research efforts are completed, the system-level group incorporates the findings into updated products for each activity. At pre-determined intervals, the system-level group captures the current state of Figure 1 activities in an internal Technical Integration Report. This report provides a comprehensive status update on the project's integration activities and also serves as a common reference for the research groups.

\section{A. Initial Research Integration Products}

The Airspace Systems Program completed an initial Technical Integration Report that captured the systems engineering methodology and provided the state of Figure 1 activities as of Fall 2007. Major highlights from this report are presented below. They include an initial demand and needs analysis; a first draft set of system-level metrics, scenario characteristics, and system-level design assumptions; and a functional modeling tool. Discussion and agreement on the system-level metrics, scenario characteristics, and assumptions still needs to occur within the research team and with the JPDO. Agreement is needed in order to realize the benefits offered by these common definitions.

Because the current projects' research had only just begun, much of the report's baseline operational, functional, and technical descriptions relied heavily on research plans and work done by prior NASA projects that are continuing in the current program. Modelers are now using these descriptions toward developing simulation systems for assessments and system-design trade studies. The integration report can also be used as a reference for researchers to see how their technologies interact in a system-wide context. It may also facilitate greater awareness of alternative concepts and technologies considered by each research area.

\section{Demand and Needs Analysis}

A preliminary demand and needs analysis assessed the ability of airports and airspace to meet the projected future demand. ${ }^{15}$ The maximum demand considered was based on a JPDO assessment that NextGen should be scalable to three times (3X) the current demand. ${ }^{16}$ Using the FAA Terminal Area Forecast, ${ }^{17}$ the AvDemand forecasting too ${ }^{18}$ generated daily flight schedules corresponding to a traffic load three times greater than a current day (2002) baseline. Then, a fast-time simulation system ${ }^{19}$ produced unconstrained trajectories for each flight and calculated the projected airport daily operations and airspace sector peak instantaneous counts. The study used these values to represent predicted airport and airspace demand (both under $3 \mathrm{X}$ conditions), respectively. For the predicted airport capacity, the study assumed a level corresponding to the FAA implementing all 2004 OEP (now known as Operational Evolution Partnership) improvements anticipated by 2015. ${ }^{20}$ Predicted airspace sector capacities were assumed to remain constant at today's monitor alert parameter levels. Demand/capacity (D/C) ratios were determined for 250 domestic airports (including 34 of 35 OEP airports) and all 730 en route sectors.

As expected, the analysis revealed that most airports and airspace sectors will be unable to meet $3 \mathrm{X}$ demand levels without capacity improvements beyond the 2015 OEP. Of the 34 OEP airports considered, 32 had predicted $\mathrm{D} / \mathrm{C}$ ratios greater than 0.7 , a value the study's authors concluded would produce significant delays. The 30 highest $\mathrm{D} / \mathrm{C}$ ratio en route sectors had predicted values between 2.5 and 6.4 .

The study considered the ability of selected airport and airspace capacity improvements to mitigate the D/C excess. Technology impacts were assessed for several potential NextGen concepts: closely-spaced parallel approaches, reduced wake vortex spacing, and ground-based automated separation assurance. Potential improvements from these concepts were based on prior results. Although the new concepts provided system-level gains, most airports and airspace sectors still exceeded their available capacities. At the airports, the new concepts reduced the OEP airports exceeding the $0.7 \mathrm{D} / \mathrm{C}$ ratio to 24 out of 34 studied. With ground-based automated separation assurance, $\mathrm{D} / \mathrm{C}$ ratios at the 30 busiest sectors were reduced to a 0.87 to 2.1 range.

The initial demand and needs analysis showed the major gaps between projected demand and system-level capacity for the nation's airports and airspace. Advanced concepts mitigated, but did not eliminate these gaps. These results suggest that numerous highly-performing, compatible ATM solutions are needed to meet the anticipated demand. Follow-on work will include system-level assessments, conducted with enhanced models, that capture the impact of integrated capacity-improving concepts studied by the Airspace Systems Program.

\section{System-Level Metrics}

Metrics provide a means to determine if the whole system or its individual parts are performing at the desired level. As architect of the NextGen system, JPDO is developing a set of system-level metrics corresponding to its system goals and objectives. ${ }^{16}$ Airspace Systems research concentrates on metrics impacting the capacity and safety goals, while remaining cognizant of the environmental protection goal. System robustness, an ability to perform consistently across a range of conditions, is given particular attention. Initial system-level metrics identified by 
NASA include those related to capacity, efficiency, and robustness. The NASA research team will work with JPDO to ensure consistent definitions.

Initial proposed system-level metrics in capacity include the daily number of flights flown, the average gate-togate block time, and the comparison of average number of flights to average delay. Efficiency metrics include total aircraft travel time for constant demand, total aircraft miles flown, and the average flight time per origin/destination pair. Robustness can be measured by comparing the system's off-nominal and nominal performance. Under nominal operations, system parameters are assumed to be within normal ranges. Off-nominal conditions imply nonnormal parameters or emergency situations that may cause adverse effects.

\section{Scenario characteristics}

Scenarios specify the configuration and underlying conditions under which proposed new concepts and technologies must operate. They reflect the anticipated NextGen environment, considering projected changes in aircraft equipage, traffic levels, flow patterns, and nominal and off-nominal operating conditions. Different levels of capacity-limiting weather conditions are also applied. Scenarios are often designed to represent limitations in today's system or potential performance-impacting events that could occur in a future environment.

The system-level group has identified scenario characteristics related to traffic and weather corresponding to nominal and off-nominal conditions. For traffic scenarios, attributes consist of flight demand including aircraft mix, schedules, and equipage; airport and airspace environment; air traffic rules; and equipment failures (as needed for off-nominal events). Weather conditions may specify airport ceiling and visibility, airport winds, winds aloft, and convective weather. Additional off-nominal events may include major outages, natural disasters, or security threats.

\section{System-Level Assumptions}

The following partial list of assumptions are applicable at the system-level. They are intended to complement those provided by the JPDO. ${ }^{2}$ Researchers investigating more detailed concepts and technologies will strive to establish their own common assumptions for conducting local and regional trade studies.

System implementation is assumed to occur consistent with the NextGen schedule. ${ }^{3}$ Different aircraft types having varying levels of avionics equipment capabilities are anticipated across the NAS. Enhanced weather prediction and communication capabilities (including Automatic Dependent Surveillance Broadcast) are assumed to be widely available.

\section{Functional Modeling Tool}

To support the functional analysis shown in Figure 1, NASA has developed a NextGen Functional Model (NFM). As a functional modeling tool, the NFM provides one means to organize and compare the design layout of different operational concepts. Researchers can use the NFM to decompose a concept into a set of functions and information links. This decomposition allows structured probing of a concept and meaningful discussion about the merits of different approaches. Diagrams showing functional relationships can help illustrate where information requirements must be specified. Understanding of dependencies between functions can be used to establish the necessary sequence of concept activities. They may also suggest possible failure modes and areas of redundancy.

To apply the NFM, researchers walk through the operational description, identifying human and machine roles and responsibilities and their associated functions. As functions are listed out, primary data input and output are identified. Researchers can then check the function and information list through a series of walk-through scenarios. These steps are applied at the level of detail needed to address the research issue under consideration.

When considering what functions to include, researchers can apply a set previously developed by the systemlevel research group. At the top system-level, these functions are broken down into "core ATM" and "enabling" categories. The top-level functions, shown in Table 1, correspond to key capabilities, functions, and enablers defined by the JPDO. ${ }^{2,3,9}$ Function numbers enable development of a tree structure with progressively greater levels of detail.

Table 1. NASA Functional Modeling Tool Top-Level Functions

\begin{tabular}{|l|c|}
\hline \multicolumn{1}{|c|}{ Core ATM Functions } & Enabling ATM Functions \\
\hline $\begin{array}{l}\text { 1 Flight Planning, Fleet Management, and } \\
\text { Collaboration }\end{array}$ & 5 Network Enabled Information Sharing \\
\hline
\end{tabular}




\begin{tabular}{|l|l|}
\hline $\begin{array}{l}\text { 2 Collaborative Air Traffic Management (includes } \\
\text { Capacity Management and Flow Contingency } \\
\text { Management) }\end{array}$ & 6 Position, Navigation, and Timing \\
\hline 3 Separation Management & 7 Surveillance \\
\hline 4 Trajectory Management & 8 Weather \\
\hline & 9 Data Management \\
\hline
\end{tabular}

In contrast with the JPDO Enterprise Architecture, ${ }^{9}$ the NFM is a design tool tailored for a research environment. It does not aim to generate a comprehensive NextGen design. It facilitates exploration of detailed technical trade spaces by supporting concept option flexibility and depth within specific areas. Functions and information flow can be specified at progressively lower levels based on research interest. Where needed, engineering judgment can be used to fill in pertinent areas not directly addressed by NASA research. An example NFM application is provided below.

\section{B. Current Research Integration Activities}

Current systems engineering efforts are focused on activities that will provide the solid foundation needed to compare technical approaches, provide needed levels of system improvement, and generate research solutions that interact effectively in NextGen. These activities lie mainly within the Systems Analysis and System Concept Assessments elements shown in Figure 1. Specific objectives include refining the system-level metrics, scenarios, and assumptions described above in close coordination with the research team and JPDO; supporting the development and distribution of more detailed metrics and scenarios that can be used to evaluate specific trade spaces; conducting system assessments of major research concepts and technologies, and conducting focused system design studies of key interfaces. As research is conducted at both the system-level and more detailed technical levels, system engineers will also update the existing operational, functional, and technical descriptions. Collectively, these activities will be captured in the next Technical Integration Report.

\section{Metrics}

Metrics development activities within the program emphasize the following areas: traceability between top-level system metrics and those used in detailed NASA research activities, impact on system-level performance, and consistent use across each applicable trade space. Traceability to system-level metrics will show how detailed technical research activities contribute directly to overall system performance. It will also allow JPDO to better assimilate NASA results with those from other research partners.

Related to capacity, the JPDO Integrated Plan emphasizes improvements in demand growth potential, transit time, predictability, and reduced susceptibility to weather and other capacity limiting events. ${ }^{16}$ Research metrics used should therefore effectively predict system-level throughput, efficiency, delay, and robustness. A 2007 Airspace Project paper by Sridhar and Swei, provides a good example of the desired traceability. ${ }^{21}$ In their studies, the authors demonstrate that a "Weather Impacted Traffic Index" (WITI) is a strong indicator of overall system delay. The WITI metric provides a means to superimpose traffic and weather conditions into a delay estimation tool.

Traffic complexity is a key measure for determining the number of aircraft that can be safely accommodated in an airspace region. ${ }^{22}$ In today's NAS, the monitor alert parameter (MAP) represents the maximum instantaneous aircraft count that a controller can manage in a given sector. Considering that future separation management functions will likely employ a greater level of automation, a complexity metric is needed that considers more factors than controller workload. Airspace project research has determined several complexity-related variables that are applicable to automation-assisted conflict resolution. ${ }^{23}$ Variables such as the available maneuvering degrees of freedom may also be applicable to completely automated conflict resolution concepts. Concept-appropriate complexity metrics should provide traceability to system-level capacity predictions and instantaneous safety assessments.

\section{Scenarios}

Common scenarios enable consistent treatment of external conditions and operating environments across a trade space. A particular area of interest focuses on scenarios representing different levels of convective weather impact on traffic delays. Researchers within the Airspace Project's traffic flow management area have used traffic and weather databases to identify historical days experiencing high, moderate, and low weather-related traffic delays. 
System-level and localized scenarios can be derived from these days and applied to other research areas within the program. High-density traffic scenarios corresponding to projected future demand are also being developed. These scenarios can be adapted to support both national and local simulation studies.

Efforts are also underway to identify and classify these and other available scenarios and create new scenarios. The system-level research group will then work to make them widely accessible across the research team and improve their compatibility with different simulation systems. Harmonization with JPDO scenarios such as those provided in the Enterprise Architecture will also be pursued. ${ }^{9}$

\section{System Assessments}

System assessments are being planned to determine the system-wide performance impact of technologies in the following research areas: capacity management, flow contingency management, and trajectory management in the terminal area and airport environment. Capabilities will generally be examined in nationwide studies using medium-fidelity simulation tools such as the Airspace Concept Evaluation System (ACES). ${ }^{19}$ The NASA VAMS Project has previously used ACES to conduct concept assessments. ${ }^{24}$ In some cases where concepts cannot be directly simulated, the national assessments may emulate results from higher fidelity local or regional studies. In these instances, specific results from prior studies may be applied to broader classes at the national level. For example, a more detailed analysis on closely-spaced parallel approaches could provide capacity input parameters for airports of similar type in a system-wide study. The assessments aim to nationalize the benefits of the combined concept elements and study their interrelationships.

In capacity management, assessments are planned that explore the interaction of dynamic airspace concepts such as high-density flow corridors (tubes) and dynamic re-sectorization to accommodate demand. Different dynamic sector partitioning methods will be considered. Flow contingency management studies will explore the efficacy of different flight delay assignment approaches based on probabilistic demand and capacity forecasts. Terminal area and airport studies will examine terminal area route strategies that fully use precise required navigation performance (RNP) routes coupled with proposed airport capacity improvements that respond to dynamic changes in demand, weather and runway configurations.

\section{System Design Studies and Functional Model Example}

Application of the iterative systems engineering process shown in Figure 1 will provide insight into design tradeoffs at the system-level and across specific sub-system interfaces. Proper consideration of these trade-offs will help ensure the overall system can meet its performance goals. Design issues concentrate on information flow and dependencies across sub-systems, timing, and functional allocation between airborne and ground systems and between humans and automation. Performance of different potential designs is evaluated using consistent capacity, safety, and environmental metrics.

A major challenge in the design of the future system is the planning and control techniques needed for high density, interdependent terminal area and airport operations. A research effort is exploring more integrated scheduling algorithms that jointly consider surface movement, runway configuration changes, arrival and departure runway balancing, and terminal area trajectory management. Tighter coupling between these activities is expected to improve throughput and efficiency. Research activities are also exploring terminal area operations known as "metroplexes" that involve interdependent operations between close-proximity major airports.

Decisions regarding allocation of functions across air-ground and human-automation systems will also play an important role in establishing the future airspace architecture and setting investment priorities. ${ }^{2,3}$ NASA is contributing to these decisions through investigation of different separation management concepts and their associated functional decompositions. Within NASA, significant prior research has demonstrated benefits for different concept solutions, however, questions concerning the comparative performance attributes of these approaches under different operating conditions remain largely unanswered. ${ }^{25,26}$ Research will focus on the potential capacity, safety, and robustness of different solutions. Contributing safety factors include areas such as data quality and availability, redundancy, and the potential for graceful degradation during sub-system failures. Benefits of centralized and distributed decision-making will also be considered. All of these traits reflect the separation management function's design capability and therefore impact system-wide performance.

The NASA NextGen Functional Model (NFM) described earlier can be applied to the separation management functional allocation problem. Researchers can use the NFM to decompose alternative concepts and compare them side-by-side. NFM representations allow researchers to describe their concepts to each other using a common format. This delineation articulates similarities and differences between approaches and can encourage helpful inquiries about research questions that may need to be considered. NFM diagrams can effectively highlight 
differences in information source and content across similar activities. Researchers can then make informed decisions about common experimental assumptions needed to ensure comparable treatment across the trade space.

Figure 2 shows the NFM applied to compare the conflict detection sub-function between two different concepts involving separation management and trajectory management. When applied in practice, written descriptions for each function and information element accompany the diagrams. In this example, a partial set of functions and information links are provided at the third level. A Unified Modeling Language (UML) ${ }^{27}$ application enables rapid changes to functions and data flows, while supporting an extensive vertical tree structure.

When using the functional diagrams to compare concepts, it is often helpful to begin with their similarities. In both concepts, a conflict detection sub-function identifies predicted losses of required separation between two aircraft. Surveillance and weather information provide supporting data. Conflict detection interfaces with a conflict resolution sub-function that creates a new trajectory around the conflict. It also notifies trajectory management functions that consider strategic trajectory changes, taking into account flight path efficiency and traffic flow management initiatives.

\section{Concept A}

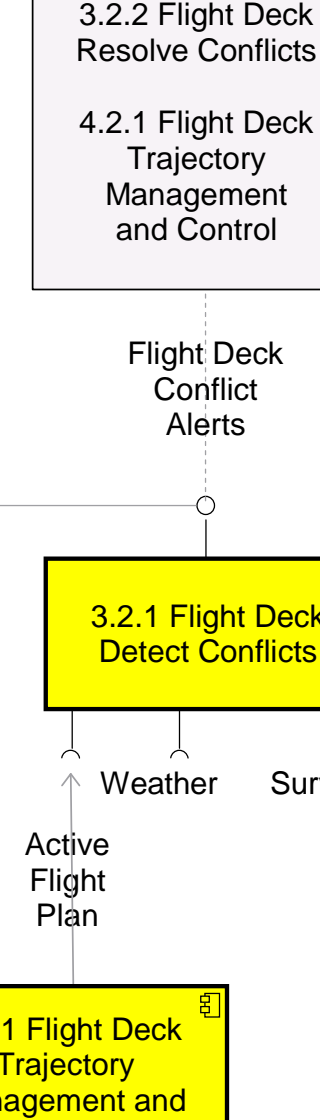

2.1 Flight Deck Trajectory Management and Control

ight Deck Conflict

Active

Flight

1 Flight Deck ement and

\section{Concept B}


Both concepts apply comparable separation management and trajectory management functions incorporating airborne and ground systems. Where these functions are performed forms a basis for distinction. Assuming normal operating conditions, conflict detection authority resides on the ground in Concept A and onboard the aircraft in Concept B. Although the diagrams provide little detail on the trajectory management functions, they suggest a more prominent role for ground-based systems in Concept A and aircraft-based systems in Concept B.

Recognition of similar activities performed by different systems can lead to interesting questions regarding airground functional allocation. Functional allocation generally considers questions about the operational conditions leading to the best system performance when viewed from different perspectives. These viewpoints include those of pilots, controllers, and the overall system.

In addition to whether conflict detection is better-handled on the ground or onboard an advanced aircraft, the diagrams can encourage other questions: How can Concept A ground-systems apply conflict resolutions that consider onboard preferences? How can aircraft flying in Concept B make informed trajectory management decisions that mitigate local or regional flow congestion?

Functional allocation decisions can also be impacted by the type of information available to different systems and the data quality. Side-by-side diagrams can help identify these considerations. Those in Figure 2 articulate a difference in the flight plan type used for conflict detection. Concept A bases conflict detection on the approved flight plan, as assigned by a ground-based system or controller. In Concept B, an onboard conflict detection system knows the active flight plan for the own aircraft, but must rely on surveillance systems for state and intent information from other aircraft. This type of assessment could lead to research studies that address the ability of airborne and ground systems to accurately predict trajectories. Each concept's susceptibility to different levels of surveillance performance (e.g. message reception probability, availability, and continuity) could also be considered. Studies assessing the benefits of these two concepts that do not directly address surveillance performance must apply common assumptions in order to ensure comparable results.

Functional analysis can also encourage broader, architectural questions. For example, some combination of Concept A and B capabilities may improve robustness to failure events, a key safety design consideration. Figure 2 provides a more detailed example for the conflict detection sub-function. A similar analysis could be conducted at the next higher level. In that case, researchers could compare the interactions between additional separation management and trajectory management sub-functions.

The NFM can assist with system allocation questions such as those described above for separation management. It can also contribute to design studies occurring across different time horizons. NASA's airspace systems design research will investigate time-based interfaces such as separation management-flow contingency management and flow contingency management-capacity management.

\section{Discussion}

Consideration of the differences between research and product development environments is important to ensure that research integration benefits are derived without overly limiting research progress or innovation. The NASA Airspace Systems Program will continue to refine its research-based application of systems engineering to achieve the proper trade-offs.

Systems engineering helps provide structure to a diverse research team chartered to provide integrated research results to the JPDO. It facilitates a consistent application of metrics, scenarios, and assumptions, thereby enabling comparative studies of different technical solutions. Methods developed also enable the assimilation of specific research products into a system-wide model that supports system-level performance assessments and design interface studies. Collectively, these activities enable the research team to analyze different trade spaces, relate specific research studies to system-wide performance, and provide ordered and well-organized contributions to JPDO research questions. This process continues iteratively as the research progresses.

These benefits notwithstanding, a systems engineering application in a research environment maintains some significant differences from the regimented approach required for product development. Research flourishes in a dynamic, uninhibited environment that allows the free flow of ideas and ability to rapidly change directions based on acquired knowledge. Product development relies on tight adherence to specified activities and schedule. Depending on the product development stage, any engineering or schedule changes may require formal processes and approvals. Research-based systems engineering therefore seeks a trade-off between structure and flexibility. It works to establish relationships between research activities and system performance, without overly constraining the research team. Understanding the unique needs of a research environment helps the research team apply the right level of structure, while maintaining necessary flexibility. 
Unlike in a development effort, system requirements for Airspace Systems research are not precisely known. JPDO is planning a scalable system that can respond to a range of demand pressures. ${ }^{16}$ Detailed metrics definitions and specific performance targets for sub-systems and components are still under consideration. Both will be subject to significant debate within the stakeholder community and will likely change over time. Dynamic targets make it difficult to determine when a proposed technology is performing at an acceptable level. Assessments considering the potential system performance impact of proposed technologies may therefore have more influence on the general research direction than on the endorsement or rejection of specific research studies.

The geographical and organizational diversity of the NASA Airspace Systems research team contributes greatly to research breadth and quality. It also presents some formidable challenges for integration. Team members span 2 NASA Centers and 35 NASA Research Announcement (NRA) awards with academia and industry. ${ }^{28}$ Organizational culture differences and variations in simulation tools and preferred metrics, scenarios, and assumptions are prevalent across the entire team. In this arena, the system-level group is working to make the team more aware of available simulation tools and complementary research activities. Ongoing dialogue about metrics, scenarios, and assumptions will strive to standardize best practices from different areas of the team, while working to establish traceability with those used by JPDO.

Systems engineering goals are most likely to be achieved when processes are easy and robust and researchers can see tangible benefits to their participation in system-level research activities. To this end, the Airspace Systems Program is working to make metrics and scenarios more broadly available through user-friendly tools and databases. System-level researchers are also collaborating with those in other research groups to determine the system-wide impact of new technologies being developed and to explore specific design questions of interest. These collaborations are aided by liaisons assigned by the system-level group to the other technical research groups. Liaisons work to become immersed in their assigned group, learning the details of proposed technical solutions. Collectively, liaison knowledge of detailed technical research can produce more informed system-level descriptions and models. Liaisons also offer a system-level perspective to the technical research groups, encouraging researchers to consider the impact of each proposed concept and technology on system-wide performance. Periodic integration workshops also provide a forum for the team to discuss the availability of common metrics and scenarios and update the status of ongoing integration-related research activities.

\section{Conclusions}

Initial demand and needs analysis results from the first iteration of systems engineering activities suggest a strong need to produce integrated research products for the 2025 NextGen system. Although combining a few promising concepts did mitigate demand and capacity gaps, these improvements did not meet JPDO goals for a NextGen system that is scalable to three times the current day capacity. These results indicate that NextGen must implement multiple advanced solutions that interact effectively.

Integrated solutions are enabled by a strong foundation of common system-level metrics, scenarios, and assumptions. The system-level research group has produced a first set of system-level metrics, assumptions, and scenario characteristics. Current work will strive to standardize these elements across the research program and ensure they are compatible with those being developed by the JPDO. Alignment of detailed metrics and scenarios with the top-level versions used by JPDO will ensure that detailed technical solutions are meeting system-level performance targets. This traceability will also help JPDO assimilate research contributions from contributing agency and industry partners.

System-wide representation of the trade space studies from operational, functional, and technical points of view enable researchers pursuing different technical approaches to compare their proposed solutions. These comparisons will motivate important feasibility questions that may impact system-level performance. They may also drive more effective architectures that leverage the combined strengths of multiple solutions.

NASA research activities are currently contributing to the next iteration of the systems engineering process. Each progressive iteration will incorporate more complete knowledge of the potential operational concepts and system design. These efforts will continue to support a broad and high-quality research portfolio that contributes to important national aviation needs.

\section{References}

\footnotetext{
${ }^{1}$ House Committee on Transportation and Infrastructure, Subcommittee on Aviation, Hearing on Airline Delays and
} 
Consumer Issues, URL: http://transportation.house.gov/hearings/hearingdetail.aspx?NewsID=300, U.S. House of Representatives, Washington, DC, 2007.

${ }^{2}$ Joint Planning and Development Office, “Concept of Operations for the Next Generation Air Transportation System,” v2.0, JPDO, Washington, DC, 2007.

${ }^{3}$ Joint Planning and Development Office, “Integrated Work Plan for the Next Generation Air Transportation System,” v0.2, JPDO, Washington, DC, Mar. 2008.

${ }^{4}$ Harvey, A., Marchand, J-L, Wagemans, H., and Vickery, K., "The Validation Data Repository: A Central Source of Validation Information for Managers and Practitioners,” Proceedings of the $21^{\text {st }}$ Digital Avionics Systems Conference, IEEE, Irvine, CA, Oct. 2002.

${ }^{5}$ Krois, P., and Rehmann J., “Air Traffic Management for Commercial and Military Systems Integrating Human Factors in Air Traffic Control Research and Acquisition,” Proceedings of the $21^{\text {st }}$ Digital Avionics Systems Conference, IEEE, Irvine, CA, Oct. 2002.

${ }^{6}$ Rehmann, J., Buondonno, K., and Humbertson, W., “The En Route Research Management Plan: A New Approach to Managing Research,” Proceedings of the $23^{\text {rd }}$ Digital Avionics Systems Conference, Salt Lake City, UT, Oct. 2004.

7 Wojcik, L., and Wang, P., "Analyzing System-Level Performance Trade-Offs Among Future Air Traffic Management Concepts," Proceedings of the $7^{\text {th }}$ Aviation Technology, Integration, and Operations Conference, AIAA-2007-7774, Belfast, UK, Sept. 2007.

${ }^{8}$ Smirti, M., and Hansen, M., “Achieving a Higher Capacity National Airspace System: An Analysis of the Virtual Airspace Modeling and Simulation Project," Proceedings of the Modeling and Simulation Technologies Conference and Exhibit, AIAA2007-6698, Hilton Head, SC, Aug. 2007.

9 Joint Planning and Development Office, "Enterprise Architecture for the Next Generation Air Transportation System," v2.0, JPDO, Jun. 2007.

${ }^{10}$ FAA, “National Airspace System System Engineering Manual,” v3.1, FAA, Washington, DC, Jun. 2006.

${ }^{11}$ Rehmann, J., Rehmann, A., and Krois, P., "Distributed Simulation: A Proposed Approach for Air Traffic Management Research," Proceedings of the 23 $3^{\text {rd }}$ Digital Avionics Systems Conference, IEEE, Salt Lake City, UT, Oct. 2004.

${ }^{12}$ Department of Defense, “Systems Engineering Fundamentals,” Defense Acquisition University Press, Belvoir, VA, Jan. 2001.

${ }^{13}$ NASA, “NASA Systems Engineering Handbook,” NASA/SP-2007-6105 Rev. 1, Dec. 2007.

${ }^{14}$ Kossiakoff, A. and Sweet, W.N., “The System Development Process,” Systems Engineering Principles and Practice, Wiley, Hoboken, NJ, 2003, pp. 50-89.

${ }^{15}$ Schleicher, D., Wendel, E., and Huang, A., “Demand Loading Analysis for a 3X NextGen Future," Proceedings of the $7^{\text {th }}$ Aviation Technology, Integration, and Operations Conference, AIAA-2007-7714, Belfast, UK, Sept. 2007.

${ }^{16}$ Joint Planning and Development Office, “Next Generation Air Transportation System Integrated Plan,” JPDO, Washington, DC, 2004.

${ }^{17}$ FAA, “Terminal Area Forecast Summary, Fiscal Years 2007-2025,” FAA, Washington, DC, 2007.

${ }^{18}$ Huang, A., Schleicher, D., and Hunter, G., "Future Flight Demand Generation Tool," Proceedings of the $4^{\text {th }}$ Aviation Technology, Integration, and Operations Conference, AIAA-2004-6400, Chicago, IL, Sept. 2004.

${ }^{19}$ Meyn, L., Windhorst, R., Roth, K., Van Drei, D., Kubat, G., Manikonda, V., Roney, S., Hunter, G., Huang, A., and Couluris, G., "Build 4 of the Airspace Concept Evaluation System," Proceedings of the AIAA Modeling and Simulation Technologies Conference and Exhibit, AIAA 2006-6110, Keystone, CO, Aug. 2006.

19 FAA, “Operational EvolutionPartnership", http://www.faa.gov/about/office_org/headquarters_offices/ato/publications/oep/FAA, Washington, DC

${ }^{21}$ Sridhar, B., and Swei, S.S.M., "Classification and Computation of Aggregate Delay Using Center-based Weather Impacted Traffic Index,” Proceedings of the $7^{\text {th }}$ Aviation Technology, Integration, and Operations Conference, AIAA-2007-7890, Belfast, UK, Sept. 2007.

${ }^{22}$ Sipe, A., Schwab, R., Haraldsdottir, A., Schoemig, E., Singleton, M., vanTulder, P., Alcabin, M., Glickman, S., and Shakarian, A., "Capacity Enhancing Air Traffic Management Concept,” AIAA/ICAS International Air And Space Symposium and Exposition: The Next 100 Years, AIAA-2003-2666, Dayton, OH, Jul. 2003.

${ }^{23}$ Kopardekar, P., Prevot, T., and Jastrzebski, M., "Traffic Complexity Measurement under Higher Levels of Automation and Higher Traffic Densities,” Proceedings of the Guidance, Navigation, and Control Conference and Exhibit, AIAA, Honolulu, HI, Aug. 2008.

${ }^{24}$ Meyn, L., Romer, T., Roth, K., Bjarke, L., and Hinton, S., "Preliminary Assessment of Future Operational Concepts Using the Airspace Concept Evaluation System," Proceedings of the $4^{\text {th }}$ Aviation Technology, Integration, and Operations Conference, AIAA-2004-6508, Chicago, IL, Sept. 2004.

${ }^{25}$ Erzberger, H., "The Automated Airspace Concept," Proceedings of the $4^{\text {th }}$ FAA/Eurocontrol ATM R\&D Seminar, FAA and Eurocontrol, Santa Fe, NM, Dec. 2001.

${ }^{26}$ Wing, D.J., Ballin, M.G., and Krishnamurthy, K., "Pilot in Command: A Feasibility Assessment of Autonomous Flight Management Operations," Proceedings of the $24^{\text {th }}$ International Congress of the Aeronautical Sciences, ICAS, Yokohama, Japan, Aug. 2004.

27 Object Management Group, “OMG Unified Modeling Language (OMG UML), Superstructure,” v2.1.2, URL: http://www.omg.org/docs/formal/07-11-02.pdf, OMG, Newton, MA, Nov. 2007. 
${ }^{28}$ NASA, “ARMD NRA Awards,” http://www.aeronautics.nasa.gov/nra_awards_airspace.htm, May 2008. 